UN ACERCAMIENTO SOCIOFONOLÓGICO DEL ESPAÑOL COLOMBIANO EN FILADELFIA, ESTADOS UNIDOS: EL CASO DE LA

VARIABLE FONOLÓGICA /S/ EN CUATRO HABLANTES DE DIFERENTES REGIONES DEL PAÍS

\title{
UN ACERCAMIENTO SOCIOFONOLÓGICO DEL ESPAÑOL COLOMBIANO EN \\ FILADELFIA, ESTADOS UNIDOS: EL CASO DE LA VARIABLE FONOLÓGICA /S/ EN CUATRO HABLANTES DE DIFERENTES REGIONES DEL PAÍS ${ }^{1}$
}

\author{
Daniel Guarín \\ Universidad del Temple (Estados Unidos) \\ guarin@temple.edu
}

Recibido: 27/01/2020 - Aprobado: 20/03/2020

DOI: doi.org/10.17533/udea.lyl.n78a07

\begin{abstract}
Resumen: Esta investigación analizó los efectos del contacto lingüístico en el español colombiano en Filadelfia (Estados Unidos) en relación con la variable fonológica /s/. Participaron cuatro hablantes de diferentes regiones del país, quienes han vivido en la ciudad por más de 15 años. Tras analizar las variaciones fonéticas, se concluyó que en el español colombiano hablado por las participantes no hay convergencia lingüística ni koineización, pues a pesar del tiempo vivido en el exterior conservan las variantes fonéticas dialectales. Además, se estableció que las hablantes muestran variación estilística según el grado de formalidad o informalidad de la situación de habla.

Palabras clave: español colombiano; variable fonológica; convergencia lingüística; koineización; español en Estados Unidos.

\section{A SOCIAL-PHONOLOGICAL APPROACH TO COLOMBIAN SPANISH IN PHILADELPHIA, UNITED STATES: THE CASE OF THE PHONOLOGICAL VARIABLE /S/ IN FOUR SPEAKERS FROM DIFFERENT REGIONS OF THE COUNTRY}

\begin{abstract}
This research analyzed the effects of language contact in Colombian Spanish in Philadelphia (United States) in relation to the phonological variable /s/. Four speakers from different regions of Colombia participated, who have lived in the city for more than 15 years. After analyzing the phonetic variations, it was concluded that there is no linguistic convergence or koineization in the Colombian Spanish spoken by the participants, because despite the time spent abroad, they preserve the dialectal phonetic variants. In addition, it was established that the speakers show stylistic variation according to the degree of formality or informality of the speech situation.
\end{abstract}

Key words: Colombian Spanish; phonological variation; language convergence; koineization; Spanish language in the United States.

1. Esta investigación se llevó a cabo durante el segundo semestre del año 2019 en Filadelfia (Estados Unidos) como proyecto para un seminario en sociolingüística de la Universidad del Temple (Estados Unidos).

LINGÜÍSTICA Y LITERATURA

163

ISSN 0120-5587

E-ISSN 2422-3174

N. ${ }^{\circ} 78,2020163-188$ 


\section{Introducción}

\subsection{Sobre la variación del español colombiano}

En Colombia existe una riquísima variación dialectal (Montes Giraldo, 1982); sus hablantes no siempre permanecen en su región dialectal de origen, sino que se desplazan por diversas razones y naturalmente conviven con hablantes de otras variedades. Con respecto a su fonología, Flórez (1963) destacó que en el español colombiano la pronunciación es en general blanda y relajada, por lo que «hay escaso impulso para mantener los sonidos finales, existe una atenuación y una diversa combinación de elementos dinámicos, tónicos y cuantitativos que dan un sello especial al español hablado por los colombianos» (p. 274). Esto, de acuerdo con el autor, genera una relajación articulatoria que logra afectar en muchos casos la precisión y la claridad del mensaje.

El presente trabajo se centró en la variación de la variable fonológica /s/ del español colombiano en cuatro hablantes de diferentes regiones de Colombia que han vivido en Filadelfia, (Estados Unidos) por más de 15 años. Las hablantes - todas mujeres - son originarias de cuatro ciudades diferentes del país: Bogotá, Manizales, Cali y Barranquilla (ver Figura 1). 


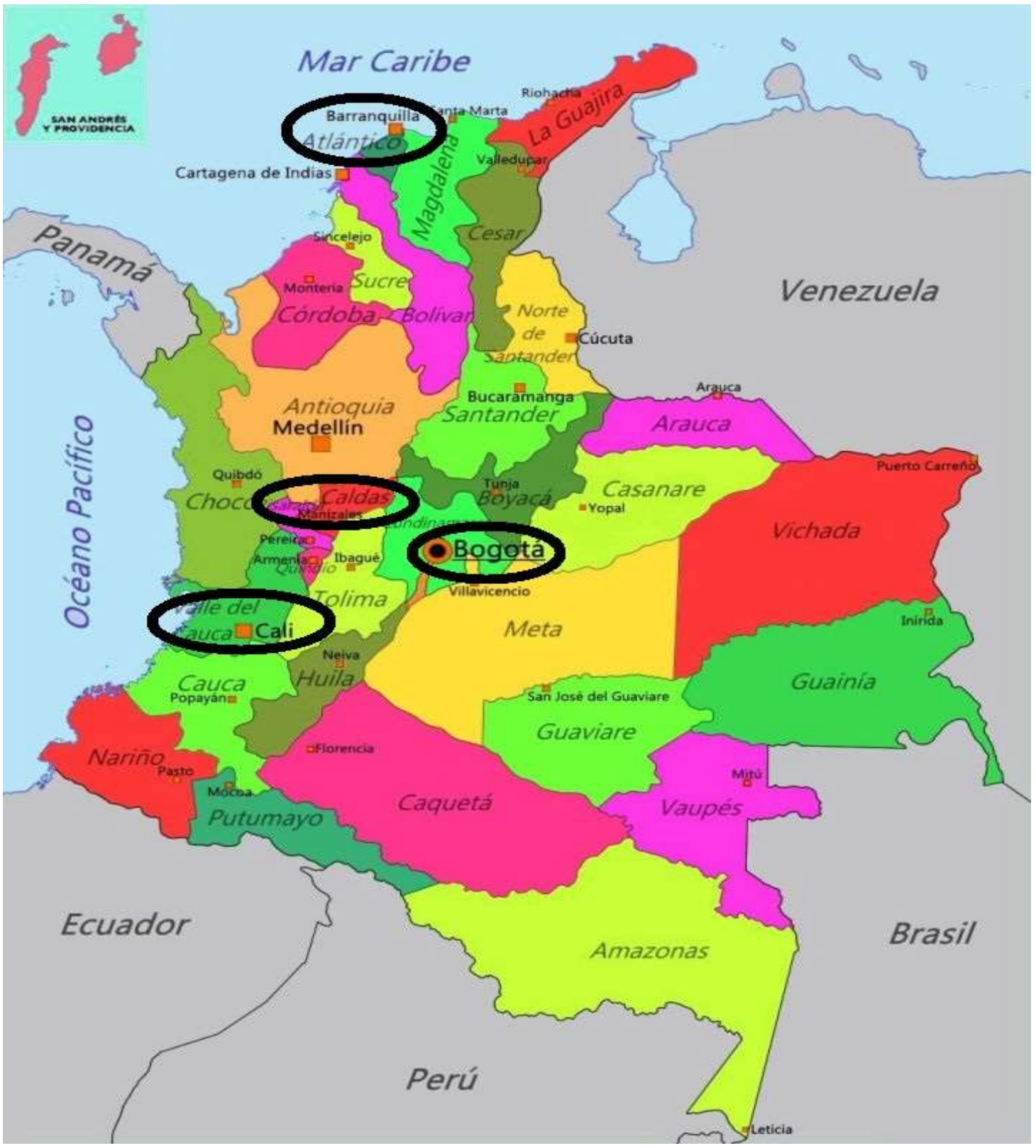

Figura 1. Ubicación geográfica de las ciudades de procedencia de las participantes en el mapa político de Colombia: Bogotá, Cali, Barranquilla y Manizales

Sobre la clasificación dialectal del español colombiano, Montes Giraldo (1982) propuso dos superdialectos, el costeño y el andino, «diferenciados por la neutralización parcial de /b/ /f/, /x/ /g/ y /r/ /1/ que se da en el primero y no en el segundo; por la aspiración de la -s y la -n velar y por algunos fenómenos gramaticales y léxicos» (pp. 51-52). Además, subdividió el dialecto 
costeño en el del pacífico y del caribe, «diferenciados principalmente por el tuteo-voseo» (p. 52), en tanto que el dialecto andino lo clasifica en centro-oriental y centro-occidental. Basándose en isoglosas léxicas, propuso otros subdialectos, «como el cartagenero, el samario, el guajiro y el costeño interior en el caribe; el antioqueño, el nariñense-caucano, el tolimense-huilense, el cundiboyacense y el santandereano en el dialecto centro-oriental» (p. 52).

A su vez, Mora et al., (2004), con base en el Atlas lingüístico-etnográfico de Colombia (ALEC), precisan las subdivisiones territoriales que se podrían encontrar dentro de los superdialectos propuestos por Montes Giraldo (1982). Apoyándonos en estas propuestas de tipología dialectal, las hablas de las informantes estudiadas para este trabajo se podrían clasificar de la siguiente manera:

\begin{tabular}{|c|c|c|c|}
\hline CIUDAD & SUBDIALECTO & DIALECTO & SUPERDIALECTO \\
\hline Bogotá & Cundiboyacense & Andino oriental & Andino \\
\hline Manizales & Antioqueño-caldense & Andino occidental & Andino \\
\hline Cali & Caucano-Valluno & Andino occidental & Andino \\
\hline Barranquilla & Atlántico & Costeño atlántico & Costeño \\
\hline
\end{tabular}

Tabla 1. Clasificación de los subdialectos, dialectos y superdialectos de las informantes en relación con las variantes dialectales del español colombiano

Con respecto a la variable /s/ y a sus variantes alofónicas, Markič (2017) comentó:

En Colombia se registran diferentes realizaciones fonéticas de /s/. En los dos dialectos costeños de Colombia, el caribe y el pacífico, conviven la aspiración (con los fenómenos concomitantes de asimilación) y la pérdida de la -s en posición de coda silábica, o posición implosiva. [...] En el superdialecto central andino, tanto occidental como oriental, normalmente se mantiene la -s implosiva y se articula como sibilante tensa. Sin embargo, Montes Giraldo señala que en materiales de habla semiespontánea recogidos en Bogotá en 1992 se manifiesta una sorprendente frecuencia de aspiración de /s/ tanto implosiva como también intervocálica (Markič, 2017, p. 188).

Por un lado, Bogotá es la capital del país y se encuentra ubicada en el centro de Colombia, en el altiplano cundiboyacense a $2640 \mathrm{~m} \mathrm{~s}$. n. m. De acuerdo con lo estipulado por Mora et al. (2004), su habla hace parte del subdialecto cundiboyacense, y la ciudad concentra el mayor 
número de habitantes; con un total de 7412 566, de acuerdo con lo reportado en el último informe de censo por el Departamento Administrativo Nacional de Estadística - DANE (2019). Igualmente, Bogotá y el país entero han experimentado grandes cambios como resultado de una situación sociopolítica convulsionada. Bogotá, como centro político y económico del país, ha atraído naturalmente migrantes de otras regiones, para quienes el habla bogotana es prestigiosa. En conexión con lo anterior, Orozco y File-Muriel (2012) señalaron:

The sociolinguistic prestige of Bogotá is quite strong, and its speech patterns have traditionally been considered a model for educated Colombians to follow. [...] this has helped maintain the prestige of Bogotano speech [...] spoken by the Colombian elite since Bogotá is home to Colombia's highest status individuals (Orozco y File-Muriel, 2012, p. 11).

Además, en la última actualización de la página web del DANE, la Dirección de Censos y Demografía indicó en el 2019 que de las 7412566 personas que actualmente habitan en Bogotá, sólo el 60,30 \% nacieron allí; por lo que es común escuchar una mezcla de dialectos y variaciones en una misma área. La variable fonológica /s/ ilustra esa variación dialectal al conservarse mayormente en posición final de sílaba en el habla cuidada de Bogotá, ya que en los estilos más informales es más frecuente su debilitamiento (Bernal Chávez \& Díaz Romero, 2017; Montes, 1996).

Por otro lado, Manizales, capital del departamento de Caldas, se encuentra ubicada en el centro occidente del país, en la región paisa - que comparte con Antioquia, Risaralda y Quindío - y hace parte del Eje Cafetero Colombiano. La ciudad se encuentra a 2200 m s. n. m. y cuenta con 434403 habitantes, según lo informado por el DANE. El habla de Manizales hace parte del subdialecto antioqueño-caldense y comparte ciertos rasgos dialectales con las demás ciudades de la región paisa, entre estos la variante apicoalveolar [s] S. Sobre esto, Canfield (1981) señala que este fonema es el mismo que se encuentra en el centro y norte de España y que es un reflejo de las variedades habladas por los colonizadores de esas regiones antes de la influencia andaluza en el siglo XVI.

De otra parte, Cali es la capital del departamento del Valle del Cauca y, de acuerdo con lo estipulado por el DANE, es la tercera ciudad más poblada de Colombia con 2471474 habitantes. Se encuentra situada en el suroccidente colombiano y tiene una altura promedio de 1 000 m s. n. m. Su habla hace parte del subdialecto caucano-valluno y varios investigadores han 
concluido que el fonema sibilante tiende a debilitarse, ya sea a través de elisión o aspiración, en todas las posiciones de sílaba y de palabra (Brown \& Brown, 2012; Espejo Olaya, 2005). Además, mencionaron Ramírez y Almira (2016) que, en el caso particular de Cali, lo que más llama la atención de los investigadores del fonema /s/ es su realización como un fono fricativo glotal sordo [h] en contextos intervocálicos e incluso en posición de ataque silábico, tanto al interior de palabra como al inicio, «siendo ese tal vez el rasgo que más identifica al habla caleña en su nivel fonético-fonológico» (Ramírez \& Almira, 2016, p. 13).

Finalmente, Barranquilla es la capital del departamento del Atlántico y se ubica sobre el margen occidental del Río Magdalena, al noroccidente del país, a 18 m s. n. m. Su habla hace parte del subdialecto costeño atlántico y tiene una población total de 1206319 habitantes, siendo así la cuarta ciudad más poblada del país. De acuerdo con Canfield (1981), uno de los rasgos más característicos del habla de la costa colombiana es la aspiración o pérdida total del fonema sibilante /s/. Así lo confirmó Espejo Olaya (2016), al señalar que la reducción del fonema sibilante se observa principalmente las zonas costeras de Colombia, fenómeno que se extiende habitualmente a los hablantes cultos. Por su parte, Bernal Chávez y Díaz Romero (2017) aseguraron que «el fonema /s/ también desaparece en posición final de palabra: mujeres [mu.'he.re], la de atrás [la.dia'tra]» (p. 27).

En síntesis, todas estas ciudades se encuentran en diferentes posiciones geográficas, cuentan con diferentes subdialectos y presentan diferentes variantes en relación con la variable fonológica /s/, como se presenta en la Tabla 2.

\begin{tabular}{|c|c|c|c|}
\hline Ciudad & Ubicación geográfica & Subdialecto & $\begin{array}{c}\text { Variante fonológica } \\
\text { característica }\end{array}$ \\
\hline Bogotá & Centro del país & Cundiboyacense & {$[\mathrm{s}],[\mathrm{h}],[\emptyset]$} \\
\hline Manizales & Centro Occidente & Antioqueño-caldense & {$[\mathrm{s}],[\mathrm{s}]$} \\
\hline Cali & Suroccidente & Caucano-valluno & {$[\mathrm{s}],[\mathrm{h}],[\emptyset]$} \\
\hline Barranquilla & Noroccidente & Atlántico & {$[\mathrm{s}],[\mathrm{h}],[\emptyset]$} \\
\hline
\end{tabular}

Tabla 2. Ubicación geográfica, subdialectos y variantes fonológicas en relación con las ciudades de origen de las participantes 


\subsection{El español en los Estados Unidos}

La presencia del español en los Estados Unidos se remonta al siglo XVI, la cual se ha visto afectada por diferentes situaciones demográficas, sociológicas y culturales (Moreno Fernández, 2006; López Morales, 2009; Hualde et al., 2010; Escobar \& Potowski, 2015). El crecimiento demográfico de la población hispana en las últimas décadas creó una oposición al uso del español por parte de ciertos sectores sociales norteamericanos, manifestada en los movimientos English Only y U.S. English que «proponen que el inglés es el idioma oficial de los Estados Unidos, con el objeto de asegurar la posición del inglés como la lengua dominante del país» (Escobar \& Potowski, p. 189).

El español, después del inglés, es la lengua más hablada en los Estados Unidos, por lo que su estudio en este contexto es actualmente uno de los principales focos de investigación (Lipski, 2008). Debido a las variaciones lingüísticas producidas por los diversos orígenes de los inmigrantes hispanohablantes y su contacto interdialectal, el español de los Estados Unidos se concibe como un mosaico de variedades (Moreno Fernández, 2009).

Por otro lado, con respecto a la distribución geográfica de la población hispana en los Estados Unidos, Lipski (2003) mencionó lo siguiente:

Las poblaciones hispanas están concentradas en el nordeste (especialmente Nueva York, Nueva Jersey, Pennsylvania y Massachusetts, junto a la ciudad de Washington), el sur (especialmente el sur de la Florida pero también Georgia y los estados vecinos) y el suroeste (Texas, Nuevo México, Arizona, California, partes de Colorado). Sólo los mexicanos y unos pequeños grupos de guatemaltecos y salvadoreños se encuentran en áreas rurales del país, donde se desenvuelven como trabajadores en las fincas agrícolas y ganaderas (Lipski, 2003, p. 232).

De esta manera, el contacto interdialectal que se genera por la confluencia de diversos factores sociolingüísticos puede llevar a la acomodación comunicativa o koineización.

La acomodación comunicativa se entiende, desde lo planteado por Giles y Ogay (2007), como el ajuste que los individuos hacen de su habla al momento de comunicarse con el fin de crear, mantener o disminuir la distancia social en la interacción. Esta acomodación, de acuerdo con Escobar y Potowski (2015), se produce cuando «los hablantes de diferentes dialectos 
comparten el mismo espacio social, suelen interactuar con cierta frecuencia en la calle, en el trabajo, en el mercado, etc.» (p. 159).

Entonces, la acomodación lingüística se produce cuando dos hablantes de diferentes dialectos entran en contacto y sus variantes lingüísticas se acomodan para reducir el distanciamiento social. Esta acomodación se puede ver reflejada en la utilización de los pronombres de segunda persona singular (tú, usted, vos) o en el uso de unidades léxicas pluriverbales, entre otros. Todo con el fin de conservar una distancia social. La koineización, por otro lado, es un fenómeno que se produce cuando los hablantes de diferentes dialectos entran en contacto y adoptan una nueva identidad lingüística en su comunidad de habla (del Valle, 1998; Johnson, 2005).

En el español de los Estados Unidos, la acomodación y la koineización han sido estudiadas por diferentes investigadores. Otheguy et al. (2007), al analizar el uso del sujeto pronominal en el español de seis comunidades hispanas en la ciudad de Nueva York, encontraron que el inglés influye en el habla de estas comunidades, y que, al estar en contacto lingüístico, la segunda generación de hablantes reconstituye una nueva comunidad de habla a la que denominan New York Spanish speech community.

Matus-Mendoza (2004) realizó una investigación en la que analiza la variación del español mexicano de Moroleón y la manera en que esta se ve desplazada al estar en contacto con otras variedades mexicanas en las granjas de champiñones de Kennett Square, (Estados Unidos). La autora señaló que «migrating to the United States inverts the prestigious patterns according to the working environment, people (...) adopt the prevailing speech forms in the workplace in the United States, where they interact» (p. 18).

Igualmente, Corredor Tapias (2018) mencionó que «las situaciones de contacto lingüístico propician la generación de fenómenos lingüísticos, como el bilingüismo, la diglosia, el contacto lingüístico y la elección de lenguas» (p. 65). Entonces, ¿cuál es la relación que tienen las diferentes variedades colombianas que se hablan en Filadelfia cuando se ponen en contacto lingüístico en un país cuya lengua principal no es el español?

Con respecto a la ausencia de un proceso de estandarización del español en los Estados Unidos, Silva-Corvalán (2000) indicó que existe una heterogeneidad lingüística que se da entre los inmigrantes de primera generación, mientras que «los hispanos nacidos en Estados Unidos 
representan una variedad de español relativamente más homogénea en el sentido de que se caracteriza por fenómenos típicos de una situación de bilingüismo intenso y extendido» (p. 14).

A su vez, Orozco (2018) aseguró que los estudios sociolingüísticos sobre el español colombiano en los Estados Unidos, específicamente en Nueva York, son escasos, lo que indica que faltaría por investigar los posibles procesos de convergencia que los dialectos colombianos atraviesan en contacto entre ellos y con otras variedades de la lengua.

Para llenar ese vacío en las investigaciones sociolingüísticas sobre el español colombiano en la diáspora, este trabajo tiene como objetivo de investigación analizar la variable fonológica /s/ en cuatro hablantes de diferentes regiones de Colombia para identificar si su habla ha tenido algún proceso de convergencia lingüística al estar en contacto con otras variedades del español en los Estados Unidos, específicamente, en Filadelfia. De esta manera, se comienza a dar una respuesta a la pregunta de investigación ¿qué efectos genera el contacto lingüístico en el español colombiano en relación con la variable fonológica /s/ en Filadelfia?

Una vez presentados los datos geográficos de las ciudades, los antecedentes de investigación concernientes a la variación del español colombiano y el objetivo de investigación, a continuación, se presentan la metodología, la discusión de resultados, la conclusión, las limitantes de la investigación y las sugerencias para investigaciones posteriores.

\section{Metodología}

El método sociolingüístico de tipo variacionista sirvió de guía en la presente investigación sobre la variación del español colombiano en Filadelfia. Silva Corvalán, (2001) sugiere una ruta metodológica de cinco pasos:

a) Observación de la comunidad e hipótesis de trabajo.

b) Selección de los hablantes.

c) Recogida de los datos.

d) Análisis de los datos:

-Análisis cualitativo:

Identificación de variables lingüísticas.

Identificación de contextos lingüísticos

Identificación de variables extralingüísticas.

Codificación de las variables

-Análisis cuantitativo: 
Cuantificación y aplicación de procedimientos estadísticos.

Interpretación de los resultados de los análisis (Silva Corvalán, 2001, p. 39).

El primer paso de este trabajo de investigación consistió en la observación de la comunidad. Para esto, se realizaron varias visitas a la calle $5^{\mathrm{a}}$ entre W. Rockland Street y W. Duncannon Av., al norte de la ciudad de Filadelfia donde hay abundancia de negocios colombianos, tales como panaderías, bares, bodegas, peluquerías y restaurantes, entre otros.

Luego de este primer acercamiento a la comunidad se logró establecer una cita para entrevistar a una de las informantes (Inf. 2), quien se encontraba en uno de los establecimientos como ayudante de operaciones. Para conseguir los demás informantes se procedió a publicar un anuncio en el grupo Colombianos en Filadelfia, de la red social Facebook, en el que se solicitaron voluntarios de nacionalidad colombiana que llevaran más de 15 años viviendo en Filadelfia. A la publicación respondieron tres informantes más que cumplían con los requisitos.

\subsection{Descripción etnográfica y social de los informantes}

La presente investigación se llevó a cabo con cuatro hablantes colombianas, todas de diferentes regiones del país y quienes llevan más de 15 años viviendo en Filadelfia, (Estados Unidos). Las entrevistas se realizaron en diferentes sectores de la ciudad, en South Philadelphia, North Philadelphia y Northeast Philadelphia, en los lugares de residencia y trabajo de las informantes.

-Informante 1: proviene de la ciudad de Bogotá, reside en Filadelfia desde hace más de 15 años y actualmente se desempeña como profesora de español en una escuela chárter. ${ }^{2}$ La entrevista se realizó en South Philadelphia. Debido a su condición de profesora de español, la informante 1 establece una comunicación bilingüe de manera constante. En inglés, lo hace con las directivas, administrativos, padres de familia y demás profesores del colegio, mientras que el español lo usa con sus estudiantes, familiares y demás profesoras de español de diferentes nacionalidades.

-Informante 2: procede de la ciudad de Manizales, lleva radicada en Filadelfia poco más de 20 años y es actualmente dueña de una empresa de limpieza de casas. En ocasiones colabora

2. Que recibe fondos del gobierno, pero opera de manera autónoma. 
con la atención de un restaurante de comida colombiana propiedad de una amiga suya. La entrevista se realizó en North Philadelphia. De acuerdo con lo expresado por la informante 2, su uso de la lengua inglesa es mínimo. A pesar de vivir en los Estados Unidos, establece una comunicación constante con colombianos de diferentes ciudades y varios latinos residentes en Filadelfia con quienes tiene lazos de amistad.

- Informante 3: nació en la ciudad de Cali, ha habitado en Filadelfia por más de 20 años y es trabajadora independiente, al realizar labores de repostería por encargo para diferentes eventos sociales. La entrevista se realizó en su hogar en Northeast Philadelphia. Según lo expresó la informante, la mayoría de sus contactos y conocidos en la ciudad son puertorriqueños (nacionalidad de su esposo), por lo que su contacto con otros colombianos es mínimo.

—Informante 4: es oriunda de la ciudad de Barranquilla, se afincó en Filadelfia hace más de 15 años y labora como profesora en una escuela de la ciudad, al igual que la informante 1. La entrevista se realizó en su lugar de trabajo en South Philadelphia. La informante es bilingüe y establece comunicación en inglés con padres de familia, directivos y amigos, mientras que con su familia en Colombia, demás profesores de español del colegio y sus estudiantes, usa el español.

La información básica relevante para el trabajo se resume en la siguiente tabla:

\begin{tabular}{|c|c|c|c|c|c|}
\hline Informante & $\begin{array}{c}\text { Lugar de } \\
\text { nacimiento }\end{array}$ & $\begin{array}{c}\text { Lugar de } \\
\text { residencia }\end{array}$ & $\begin{array}{c}\text { Tiempo en } \\
\text { EE. UU. }\end{array}$ & Ocupación & Edad \\
\hline Inf. 1 & Bogotá & Filadelfia, & +15 años & $\begin{array}{c}\text { Profesora de español en } \\
\text { escuela primaria }\end{array}$ & 36 \\
\hline Inf. 2 & Manizales & Filadelfia, & +20 años & $\begin{array}{c}\text { Trabajadora independiente. } \\
\text { Tiene su propio negocio de } \\
\text { limpieza de casas. }\end{array}$ & 55 \\
\hline Inf. 3 & Cali & Filadelfia, & +20 años & $\begin{array}{c}\text { Trabajadora independiente. } \\
\text { Tiene su propio negocio de } \\
\text { tortas para eventos }\end{array}$ & 59 \\
& Barranquilla & Filadelfia, & +15 años & $\begin{array}{c}\text { Profesora de español en } \\
\text { escuela primaria }\end{array}$ & 31 \\
\hline Inf. 4 & PA & & PA & & \\
\hline
\end{tabular}

Tabla 3. Relación de los datos personales sobre las informantes 


\subsection{Técnicas de recolección de datos}

Como herramienta de recolección de datos se empleó la entrevista sociolingüística semidirigida, «en la cual se produce una conversación acerca de los aspectos relacionados con la vida del entrevistado en diferentes etapas de su vida, aspectos de la vida de la comunidad y experiencias en general del entrevistado» (Díaz Campos, 2014). De acuerdo con Labov (1972), «el propósito de la investigación lingüística en una comunidad debe ser descubrir cómo habla la gente cuando no está siendo observada sistemáticamente; no obstante, solo podemos obtener dicha información mediante observación sistemática» (p. 209), esto se conoce como la «paradoja del observador». Con el fin de reducir a un mínimo posible los efectos de dicha paradoja en el habla natural de las informantes, antes de comenzar con las entrevistas, se realizó una breve conversación informal y se intentó entrar en confianza con las informantes al hablar sobre temas en común, tales como los gustos culinarios, la vida en Colombia antes de la inmigración y las experiencias en los Estados Unidos.

Luego, se realizó una entrevista informal semidirigida, en la que se abordaron diferentes temas de su vida cotidiana y personal, como el trabajo, la familia, las actividades de ocio, las sensaciones de vivir en el exterior, las motivaciones para dejar Colombia, las percepciones sobre la nueva ciudad, sus recuerdos de sus orígenes y sus planes para el futuro. Cada entrevista duró aproximadamente 15 minutos.

Al finalizar las entrevistas, se les solicitó a las informantes que leyeran un extracto del cuento La señorita del silencio $S$., con el objetivo de obtener un estilo más cuidado y establecer un contraste frente al estilo más informal que se dio durante la entrevista, pues de acuerdo con Silva- Corvalán (2000), los hablantes muestran variación estilística que abarca desde el registro formal al vernáculo según los factores sociales y contextuales. Debido a esto, Díaz Campos (2014) sugirió incluir, además de la entrevista, «la lectura de un párrafo, de una lista de palabras y de una lista de pares mínimos» (p. 12). De este modo, durante la investigación sólo se realizó la entrevista y la lectura del cuento. ${ }^{3}$

3. Siguiendo el consejo de uno de los evaluadores del artículo, se decidió añadir la lectura de lista de palabras, por lo que se volvió a contactar a las informantes para que realizaran un último registro. Sin embargo, debido al 
Con respecto a la metodología de recolección de datos se siguió lo sugerido por Feagin (2013) en cuanto a los recursos de grabación: «it is crucial that researchers use recording equipment meeting the technical specifications needed to produce sound of high enough quality [...] Also crucial is selection of recording location. Clearly, quiet locations are better than noisy ones» (p. 23). Para las grabaciones se utilizó una grabadora de voz Olympus WS-853.

Por cada informante se analizaron, aproximadamente, 100 tokens durante la entrevista y 64 tokens durante la lectura con la variable fonológica /s/ y sus variantes en posición inicial de palabra, posición intervocálica, posición de coda, y posición final de palabra (ver Tabla 4).

\begin{tabular}{|c|c|c|c|}
\hline Informante & $\begin{array}{c}\text { Tokens en } \\
\text { entrevista }\end{array}$ & $\begin{array}{c}\text { Tokens en } \\
\text { lectura }\end{array}$ & $\begin{array}{c}\text { Total de } \\
\text { tokens }\end{array}$ \\
\hline Inf. 1 (Bogotá) & 97 & 64 & 161 \\
\hline Inf. 2 (Manizales) & 108 & 64 & 172 \\
\hline Inf. 3 (Cali) & 90 & 64 & 154 \\
\hline Inf. 4 (Barranquilla) & 104 & 64 & 168 \\
\hline
\end{tabular}

Tabla 4. Relación de la cantidad de tokens analizados en la investigación

\section{Resultados}

El objetivo de este trabajo de investigación consistió en analizar la variable fonológica /s/ en el habla de cuatro informantes de diferentes regiones de Colombia que residen actualmente en Filadelfia, (Estados Unidos). En la Tabla 5 se presentan la variable y las variantes encontradas tras el análisis de datos.

\begin{tabular}{|c|c|c|c|}
\hline \multicolumn{4}{|c|}{ Variable=/s/ } \\
\hline Variante & Entrevista & Lectura & Total \\
\hline Variante sibilante alveolar [s] & 306 & 247 & 553 \\
\hline
\end{tabular}

COVID-19 y los problemas de salubridad presentados en la ciudad de Filadelfia, este último registro no se pudo llevar a cabo. 
UN ACERCAMIENTO SOCIOFONOLÓGICO DEL ESPAÑOL COLOMBIANO EN FILADELFIA, ESTADOS UNIDOS: EL CASO DE LA VARIABLE FONOLÓGICA /S/ EN CUATRO HABLANTES DE DIFERENTES REGIONES DEL PAÍS

\begin{tabular}{|c|c|c|c|}
\hline Variante aspirada [h] & 55 & 2 & 57 \\
\hline Variante apicoalveolar [s] & 21 & 7 & 28 \\
\hline Variante elidida [Ø] & 17 & 0 & 17 \\
\hline
\end{tabular}

Tabla 5. Distribución de las variantes fonológicas en la entrevista y en la lectura del texto

De acuerdo con la Tabla 5, se obtuvieron cuatro variantes de la variable fonológica /s/ en el español colombiano en Filadelfia. La variante sibilante alveolar [s] es la más frecuente, con un total de 553 tokens. En segundo lugar, se encuentra la variante aspirada [h], con un total de 57 tokens; cabe resaltar, sin embargo, que las apariciones de esta variante disminuyeron notablemente al pasar del habla más informal de la entrevista al habla más formal de la lectura, con apenas 2 casos de realización. En tercer lugar se ubica la variante apicoalveolar [s] de de la cual se obtuvieron 28 tokens. En cuarto lugar aparece la variante elidida [Ø], con un total de 17 apariciones y nuevamente una notable reducción de la variante durante la lectura. A continuación, se presenta un análisis de datos de los resultados obtenidos por cada hablante.

\subsection{Resultados y análisis de datos de la informante de Bogotá}

La entrevista con la informante de Bogotá duró aproximadamente 20 minutos. El tema principal de la conversación fue su experiencia como profesora de español en los Estados Unidos, pues la participante afirmó que su formación académica es como psicóloga, no como pedagoga.

Del registro de la entrevista se analizaron 97 tokens, todos con la variante [s]:

/S/oy de Bogotá como le/s/ conté, llegué aquí ha/s/e die/s/i/s/éi/s/, die/s/i/s/iete año/s/, yo creo. Mi objetivo original era aprender inglé/s/ porque Yo había e/s/tudiado alguna/s/ co/s/ita/s/ en el Colombo.

Mi profe/s/ión, yo /s/oy p/s/icóloga de profe/s/ión. Llegaba a los pro/s/e/s/o/s/ má/s/ alto/s/ y me sacaban porque no hablaba nada de /s/egunda lengua.

$\mathrm{La} / \mathbf{s} / \mathrm{mae} / \mathbf{s} / \mathrm{tra} / \mathbf{s} / / \mathbf{s} / \mathrm{omo} / \mathrm{s} /$ muy territoriale/s/. Y $/ \mathbf{s} / \mathrm{i}$ tu ere/s/ una buena mae/s/tra te re/s/petábamo/s/. Pero $/ \mathbf{s} / \mathbf{i}$ tu no era/s/ una buena mae/s/tra te mirábamo/s/ y te mirábamo/s/ y te mirábamo/s/.

No/s/ entrenaban un año, veíamo/s/ a la/s/ demá/s/ mae/s/tra/s/. mi/s/ clase/s/ eran /s/uper aburrida/s/ y lo/s/ niño/s/ —eeh—, no /s/oportaban. Entonce/s/ yo gritaba mi alma y no ha/s/ían ca/s/o. 
De otro lado, durante la lectura del cuento, se registraron 64 tokens, todos con la variante [s]:

Un día llegó un /s/irco al paí/s/ de la/s/ letra/s/. El primer día fue gente a ver el /s/irco, pero no dema/s/iada. Al día /s/iguiente fue meno/s/ gente y al /s/iguiente meno/s/. El director del /s/irco pensó que /s/ería /s/u ruina a/s/í que organi/s/ó un de/s/file para que todo el mundo $/ \mathbf{s} /$ upiera cuanta/s/ co/s/as divertida/s/ tenían en el /s/irco y fue/s/en a verlo.

Organi/s/aron un de/s/file lleno de color y de mú/s/ica. La/s/ trompeta/s/ iban adelante, lo/s/ tambore/s/ detrá/s/, le /s/eguían lo/s/ platillo/s/. Como /s/i no fuera ba/s/tante, un empleado del /s/irco ve/s/tido de manera extravagante gritaba todo lo que podía di/s/iendo vengan, /s/eñore/s/, vengan a ver el maravillo/s/o /s/irco rojo-a/s/ul. Podrán contemplar leone/s/, tigre/s/, elefante/s/ gigante/s/co/s/, foca/s/amae/s/trada/s/, perro/s/ equilibri/s/ta/s/, divertido/s/ paya/s/o/s/, arrie/s/gado/s/ trape/s/ista/s/.

A continuación, se presenta la tabulación de las variantes:

\begin{tabular}{|c|c|c|}
\hline $\begin{array}{c}\text { Informante 1 } \\
\text { (Bogotá) }\end{array}$ & {$[\mathbf{s}]$} & Tokens totales \\
\hline Entrevista & 97 tokens & 97 tokens \\
& $(100 \%)$ & $(100 \%)$ \\
\hline $\begin{array}{c}\text { Lectura de } \\
\text { texto }\end{array}$ & 64 tokens & 64 tokens \\
$(100 \%)$ & $(100 \%)$ \\
\hline
\end{tabular}

Tabla 6. Relación de la variante obtenida de la informante de Bogotá

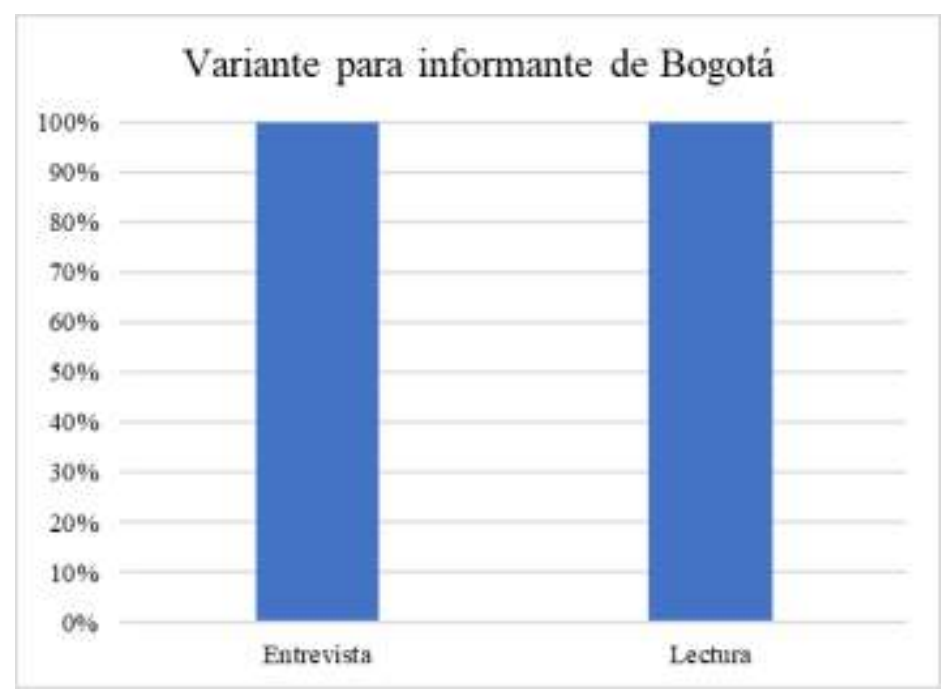

Gráfico 1. Relación de la variante obtenida de la informante de Bogotá 
Estos registros permiten tener una perspectiva global sobre el uso de sibilantes en el habla bogotana en el cual se utiliza la variante [s] de la misma manera en posición de coda y posición intervocálica, tanto en el registro informal como en el formal.

\subsection{Resultados y análisis de datos de la informante de Manizales}

La entrevista con la informante de Manizales duró aproximadamente 15 minutos. El tema principal de la conversación fueron sus viajes por el mundo, especialmente sobre lo que más disfruta de sus visitas a Colombia, especialmente sobre su último viaje, en el que recorrió el departamento del Quindío — región de procedencia del entrevistador, lo que facilitó una conversación más amena y en confianza-; y sus planes para el futuro.

De la entrevista se obtuvieron 108 tokens: 90 con la variante [s], 17 con la variante [s] y 1 con la variante $[\mathbf{h}]$ :

Ayer ju/s/tamente cumplí 27 año/s/ de haber llegado a lo/s/ E/s/tado/s/ Unido/s/. No ha /s/ido muy fá/s/il al prin/s/ipio por el idioma má/s/ que todo.

E/s/ como volver a na/s/er. Llega/s/ a un paí/s/ donde no /s/abe/s/ leer, no /s/abe/s/ escribir, no te sabe/s/ ni /s/ubir a un bu/s/, no conoce/s/ nada.

Entonce/s/ fue complicado mucho. Pero /s/in embargo pue/s/ como venía con mucha/s/ e/s/pectativa/s/, con /s/ueño/s/ qué cumplir, meta/s/ y pue/s/ e/s/a fue una de la/s/ razone/s/ de llegar a e/s/te paí/s/ maravillo/s/o que aparte de e/s/o me adoptó porque /s/eguí trabajando fuertemente para lograr lo que quería y pue/s/ creo que ya e/s/toy terminando todo/s/ mi $/ \mathbf{s} / / \mathbf{s} / \mathbf{u e n ̃ o} / \mathbf{s} /$.

Por otra parte, con respecto al ejercicio de lectura, se analizaron 64 tokens: 57 con la variante sibilante [s] y 7 con la variante apicoalveolar [s]:

Un día llegó un /s/irco al paí/s/ de la/s/ letra/s/. El primer día fue gente a ver el /s/irco, pero no dema/s/iada. Al día /s/iguiente fue meno/s/ gente y al /s/iguiente meno/s/. El director del /s/irco pensó que $/ \mathrm{s} / \mathrm{ería} / \mathrm{s} / \mathrm{u}$ ruina a/s/í que organi/s/ó un de/s/file para que todo el mundo $/ \mathrm{s} /$ upiera cuanta/s/ co/s/as divertida/s/ tenían en el /s/irco y fue/s/en a verlo.

Organi/s/aron un de/s/file lleno de color y de mú/s/ica. La/s/ trompeta/s/ iban adelante, lo/s/ tambore/s/ detrá/s/, le /s/eguían lo/s/ platillo/s/. Como /s/i no fuera ba/s/tante, un empleado del /s/irco $\mathrm{ve} / \mathrm{s} /$ tido de manera extravagante gritaba todo lo que podía di/s/iendo vengan, /s/eñore/s/, vengan a ver 
el maravillo/s/o /s/irco rojo-a/s/ul. Podrán contemplar leone/s/, tigre/s/, elefante/s/ gigante/s/co/s/, foca/s/ amae/s/trada/s/, perro/s/ equilibri/s/ta/s/, divertido/s/ paya/s/o/s/, arrie/s/gado/s/ trape/s/ista/s/.

A continuación, se presenta la tabulación de las variantes para la informante de Manizales:

\begin{tabular}{|c|c|c|c|c|}
\hline $\begin{array}{c}\text { Informante 2 } \\
\text { (Manizales) }\end{array}$ & Variante [s] & Variante [s] & Variante [h] & $\begin{array}{c}\text { Tokens } \\
\text { totales }\end{array}$ \\
\hline Entrevista & $\begin{array}{c}90 \\
(83.33 \%)\end{array}$ & $\begin{array}{c}17 \\
(15.74 \%)\end{array}$ & $\begin{array}{c}1 \\
(0.93 \%)\end{array}$ & $\begin{array}{c}108 \\
(100 \%)\end{array}$ \\
\hline $\begin{array}{c}\text { Lectura de } \\
\text { texto }\end{array}$ & $\begin{array}{c}57 \\
(89.06 \%)\end{array}$ & $\begin{array}{c}(10.94 \%) \\
(0 \%)\end{array}$ & $\begin{array}{c}64 \\
(100 \%)\end{array}$ \\
\hline
\end{tabular}

Tabla 7. Relación de las variantes obtenidas de la informante de Manizales

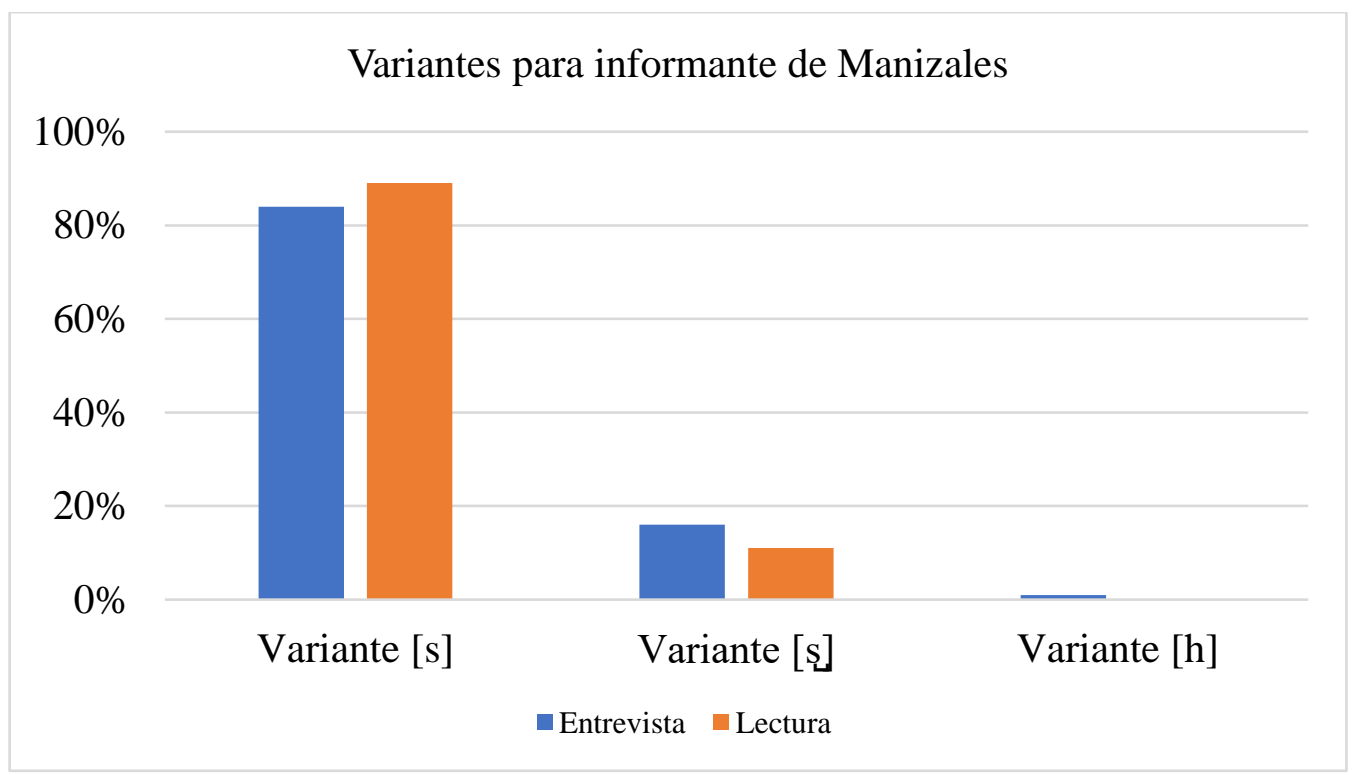

Gráfico 2. Relación de las variantes obtenidas de la informante de Manizales

Los datos tomados corroboran lo afirmado por Canfield (1981), pues demuestran que la variante apicoalveolar es característica de esta región y se conserva en el habla inmigrante a 
pesar del tiempo de permanencia por fuera del país y del contacto lingüístico con otras variedades lingüísticas en Filadelfia. Cabe resaltar, además, que durante el ejercicio de lectura en el que el habla tiende a ser más formal y cuidada, el uso de esta variante se conserva. Sin embargo, no se puede descartar la posibilidad de que la cercanía dialectal con el entrevistador haya impulsado la frecuencia de uso de la variante apicoalveolar.

\subsection{Resultados y análisis de datos de la informante de Cali}

La entrevista con la informante de Cali duró aproximadamente 20 minutos. El tema de conversación giró en torno a su vida en Filadelfia y, en gran parte, sobre pasteles - mientras enseñaba fotos de sus creaciones reposteras - y los comentarios de sus clientes al probarlos. Este tema de conversación resultó ser de gran ayuda, pues cuanto más hablaba la informante sobre sus productos, más emocionada y tranquila se mostraba con la entrevista, lo que facilitó la obtención de un registro de habla informal.

De la entrevista informal se obtuvieron 90 tokens. 64 con la variante [s], 14 con la aspirada [h], 8 con la elidida $[\varnothing]$ y 4 con la apicoalveolar [s]:

Me adapté rapidí/s/imo.

$\mathrm{O} / \mathbf{s} /$ ea, en Colombia e/s/tamo/Ø/ muy, eh ¿cómo e/s/? Que /s/iempre e/s/tamo/h/ pendiente/Ø/ que no/s/ critican, que $/ \mathbf{s} / \mathbf{i} \mathrm{ha} / \mathrm{s} / \mathrm{emo} / \mathbf{h} / \mathrm{e} / \mathrm{s} /$ to la gente lo va a ver y que $/ \mathbf{s} / \mathrm{iempre} \mathrm{e} / \mathbf{s} / \mathrm{tamo} / \emptyset / \mathrm{di} / \mathbf{s} / \mathrm{iendo}$, qué opina la gente, qué di/s/e la gente.

A mí no me gu/s/ta mucho la /s/iudad. E/s/ /s/u/s/ia. /S/iempre hay grafiti/Ø/. La/s/ parede/Ø/ e/s/crita/s/. E/s/o no me gu/s/ta. ¿Qué má/s/?

Me va muy bien, fíjate. Como te digo lo empe/s/é ha/s/iendo como un hobby porque e/h/ algo que me apa/s/iona. /S/iempre me ha gu/s/tado la repo/s/tería. Enton/s/e/s/ una ve/s/ que tuve mi/s/ hija/s/ e/s/tuve libre /s/in trabajar porque mi e/s/po/s/o me dijo «ay, quédate en la ca/s/a cuidando la/h/ niña/s/», pero yo no qui/s/e quedarme $/ \mathbf{h} /$ olamente en $\mathrm{mi} \mathrm{ca/s/a}$.

A su vez, durante el ejercicio de lectura se registraron 64 tokens, todos con la misma variante sibilate $[\mathrm{s}]$ :

Un día llegó un /s/irco al paí/s/ de la/s/ letra/s/. El primer día fue gente a ver el /s/irco, pero no dema/s/iada. Al día /s/iguiente fue meno/s/ gente y al /s/iguiente meno/s/. El director del /s/irco pensó 
que /s/ería /s/u ruina a/s/í que organi/s/ó un de/s/file para que todo el mundo /s/upiera cuanta/s/ co/s/as divertida/s/ tenían en el /s/irco y fue/s/en a verlo.

Organi/s/aron un de/s/file lleno de color y de mú/s/ica. La/s/ trompeta/s/ iban adelante, lo/s/ tambore/s/ detrá/s/, le /s/eguían lo/s/ platillo/s/. Como /s/i no fuera ba/s/tante, un empleado del /s/irco ve/s/tido de manera extravagante gritaba todo lo que podía di/s/iendo vengan, /s/eñore/s/, vengan a ver el maravillo/s/o /s/irco rojo-a/s/ul. Podrán contemplar leone/s/, tigre/s/, elefante/s/ gigante/s/co/s/, foca/s/ amae/s/trada/s/, perro/s/ equilibri/s/ta/s/, divertido/s/ paya/s/o/s/, arrie/s/gado/s/ trape/s/ista/s/.

A continuación, se presenta la tabulación de las variantes para la informante 3:

\begin{tabular}{|c|c|c|c|c|c|}
\hline $\begin{array}{c}\text { Informante 3 } \\
\text { (Cali) }\end{array}$ & $\begin{array}{c}\text { Variante } \\
\text { [s] }\end{array}$ & $\begin{array}{c}\text { Variante } \\
\text { [h] }\end{array}$ & $\begin{array}{c}\text { Variante } \\
\text { [s] }\end{array}$ & $\begin{array}{c}\text { Variante } \\
{[\boldsymbol{\emptyset}]}\end{array}$ & Total \\
\hline Entrevista & 64 & $\begin{array}{c}14 \\
(71.11 \%)\end{array}$ & $\begin{array}{c}4 \\
(15.56 \%)\end{array}$ & $\begin{array}{c}8 \\
(4.44 \%)\end{array}$ & $\begin{array}{c}90 \\
(100 \%)\end{array}$ \\
\hline $\begin{array}{c}\text { Lectura de } \\
\text { texto }\end{array}$ & $\begin{array}{c}64 \\
(100 \%)\end{array}$ & $\begin{array}{c}(0 \%) \\
(0 \%)\end{array}$ & $(0 \%)$ & $\begin{array}{c}64 \\
(100 \%)\end{array}$ \\
\hline
\end{tabular}

Tabla 8. Relación de las variantes obtenidas de la informante de Cali

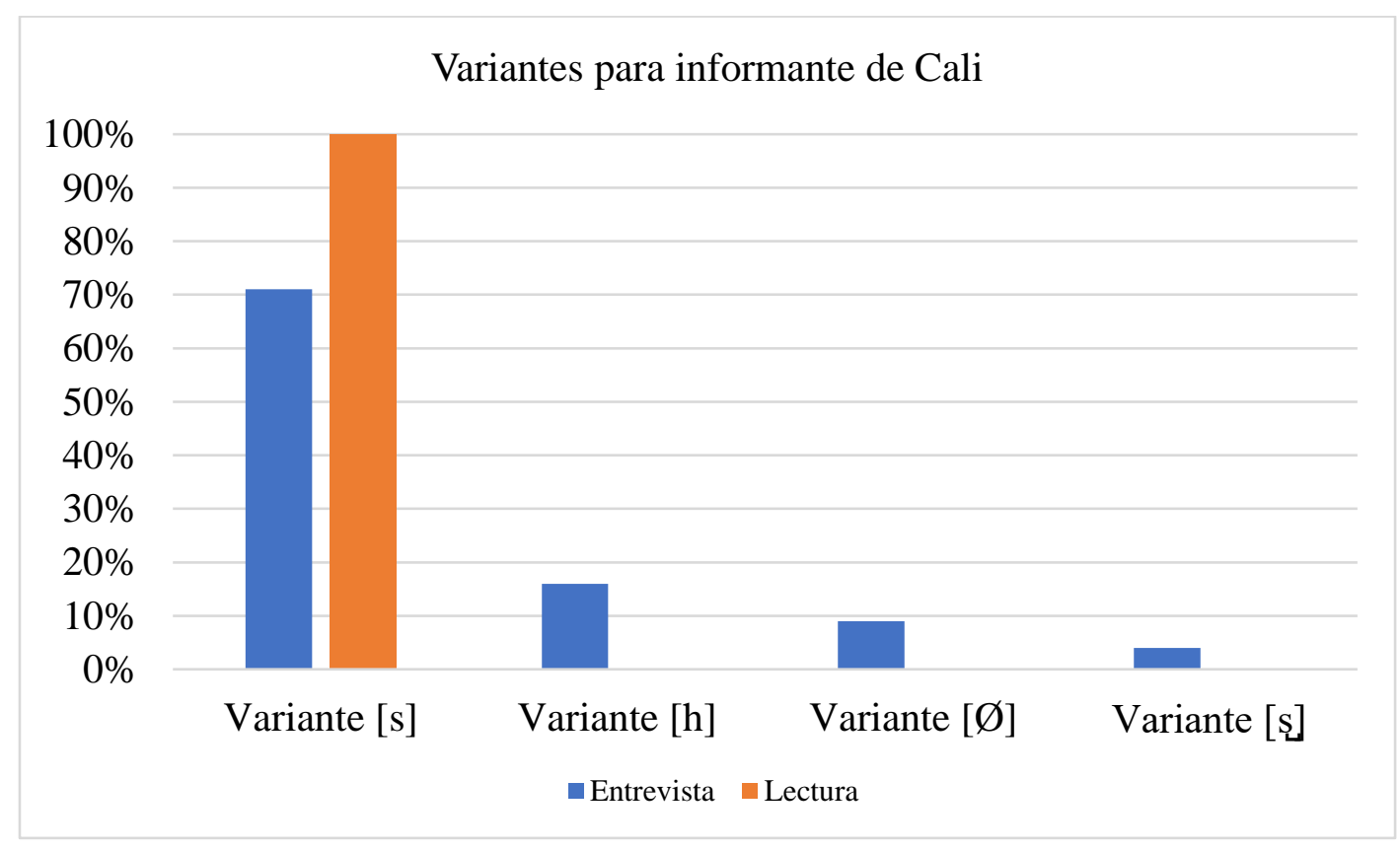

Gráfico 3. Relación de las variantes obtenidas de la informante de Cali 
Los datos indican que la informante de Cali en su la entrevista presentó las tres variantes de la variable /s/; sin embargo, cuando se realizó el ejercicio de lectura, todas las variantes se omitieron, menos la variante [s], lo que indicaría que la informante de Cali percibe el uso de la sibilante como un registro de habla de prestigio.

\subsection{Resultados y análisis de datos de la informante de Barranquilla}

La entrevista con la informante de Barranquilla duró aproximadamente 15 minutos. El tema principal de la conversación fue su experiencia como profesora de español en los Estados Unidos y sus proyectos académicos de inmersión para los niños de la escuela, a quienes lleva a países latinoamericanos - entre ellos Colombia - con el fin de que tengan una inmersión lingüística y cultural dentro de la lengua española.

De la entrevista se obtuvieron 104 tokens: 55 con la variante [s], 40 con la variante aspirada [h] y 9 con la variante elidida [Ø]:

La e/s/cuela tiene un programa donde llevamo/s/ a niño/h/ de /s/éptimo grado por una /s/emana a hacer trabajo /s/ocial.

Empe/s/amo/h/ con Ecuador, luego agregamo/h/ Nicaragua, ahora agregamo/h/ Colombia y el año $\mathrm{pa} / \mathrm{s} /$ ado agregamo/Ø/ Guatemala.

Entonce/s/ cada año llevamo/h/ má/h/ o meno/s/ el /s/e/s/enta por/s/iento de lo/s/ niño/Ø/ de /s/éptimo grado a e/h/ta, e/h/te trabajo y por lo meno/h/ e/h/te año vamo/h/ a lo/s/ tre/Ø/ paí/s/e/h/.

Con respecto al ejercicio de lectura, se analizaron 64 tokens: 62 con la variante [s] y 2 con la variante aspirada $[\mathbf{h}]$ :

Un día llegó un /s/irco al paí/s/ de la/s/ letra/s/. El primer día fue gente a ver el /s/irco, pero no dema/s/iada. Al día /s/iguiente fue meno/s/ gente y al /s/iguiente meno/s/. El director del $/ \mathbf{s} /$ irco pen/s/ó que /s/ería /s/u ruina a/s/í que organi/s/ó un de/h/file para que todo el mundo /s/upiera cuanta/s/ co/s/as divertida/s/ tenían en el /s/irco y fue/s/en a verlo.

Organi/s/aron un de/h/file lleno de color y de mú/s/ica. La/s/ trompeta/s/ iban adelante, lo/s/ tambore/s/ detrá/s/, le /s/eguían lo/s/ platillo/s/. Como /s/i no fuera ba/s/tante, un empleado del /s/irco ve/s/tido de manera extravagante gritaba todo lo que podía di/s/iendo vengan, /s/eñore/s/, vengan a ver el maravillo/s/o /s/irco rojo-a/s/ul. Podrán contemplar leone/s/, tigre/s/, elefante/s/ gigante/s/co/s/, foca/s/ amae/s/trada/s/, perro/s/ equilibri/s/ta/s/, divertido/s/ paya/s/o/s/, arrie/s/gado/s/ trape/s/ista/s/.

A continuación, se presenta la tabulación de las variantes de la informante de Barranquilla: 


\begin{tabular}{|c|c|c|c|c|}
\hline Informante 4 & Variante [s] & Variante [h] & Variante [Ø] & Total \\
(Barranquilla) & & 40 & 9 & 104 \\
\hline Entrevista & 55 & $(38.46 \%)$ & $(8.65 \%)$ & $(100 \%)$ \\
\hline Lectura de & $62.88 \%)$ & 2 & 0 & 64 \\
textos & $(96.88 \%)$ & $(3.13 \%)$ & $(0 \%)$ & $(100 \%)$ \\
\hline
\end{tabular}

Tabla 9. Relación de las variantes obtenidas de la informante de Barranquilla

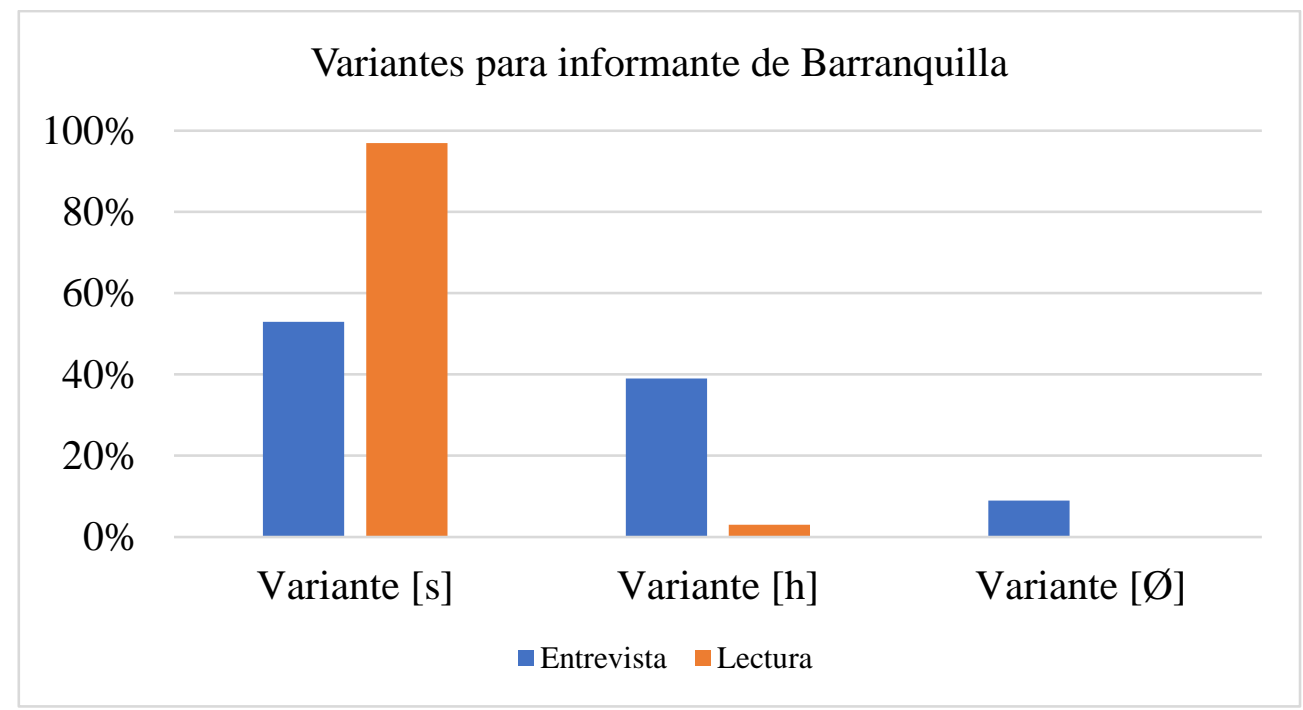

Gráfico 4. Relación de las variantes obtenidas de la informante de Barranquilla

Los tokens obtenidos a partir de la entrevista y la lectura de la informante de Barranquilla muestran una variación estilística. Mientras que en la entrevista se identifican variantes elididas y aspiradas, en su mayoría, durante la lectura de texto, cuando su estilo fue cuidado, estas variantes desaparecieron casi por completo y se hizo uso casi total de la sibilante [s], lo que indicaría cierta idea de prestigio en relación con la variante sibilante.

Una vez compartidos los resultados de la investigación se procede, a continuación, a compartir las conclusiones del trabajo. 


\section{Conclusiones}

Como se estableció desde un principio, el presente trabajo tuvo como objetivo dar respuesta a la siguiente pregunta: ¿qué efectos genera el contacto lingüístico en el español colombiano en Filadelfia en relación con la variable fonológica /s/?

De acuerdo con los antecedentes de la investigación, en el habla bogotana la variable /s/ se conserva en el registro informal, pero tiende a perderse en los niveles socioculturales bajos; a su vez, el habla de Manizales se caracteriza por el uso de la variante apicoalveolar; en Cali, la variable /s/ tiende a aspirarse o a elidirse; y en Barranquilla la elisión de la /s/ es habitual incluso en hablantes de niveles educativos altos.

Estas generalidades prototípicas de las cuatro regiones dialectales de Colombia se asemejan con los resultados obtenidos en el análisis cualitativo de las variantes en el habla informal de cuatro informantes residentes en Filadelfia, (Estados Unidos) y oriundas de las 4 regiones descritas en esta investigación. Por esta razón, se puede señalar que, a pesar del tiempo que las informantes han vivido en los Estados Unidos y del contacto lingüístico con el inglés y otros dialectos del español, sus dialectos regionales se conservan, por lo que es posible concluir que el proceso de convergencia o koineización no se da en el español colombiano de Filadelfia, dentro de las limitaciones de este estudio y en base a lo que los datos sugieren.

Además, se puede concluir que, en efecto, como lo mencionó Silva-Corvalán (2001), las informantes de Cali y Barranquilla son multidialectales, pues su habla informal se dio durante la entrevista, resaltando en ella el uso de variantes aspiradas o elididas, mientras que, durante la lectura, al hacer uso de su registro cuidado o formal, omiten las mencionadas variantes y tienden a emplear la variante sibilante [s], característica del habla bogotana.

La informante de Manizales, sin embargo, conserva su uso de la variante apicoalveolar [s] tanto en su habla informal como en su ejercicio de lectura, lo que podría interpretarse como una marca de prestigio regional. Similar a lo encontrado por Labov (1972) en su estudio de la variación dialectal de Martha s Vineyard y por Rivera-Mills (2011) en relación con el voseo y la identidad de hondureños y salvadoreños en el oeste de los Estados Unidos, en el cual menciona que «it is possible that this linguistic feature will continue to be a transnational feature that will 
be integrated into the many varieties of Spanish in the U.S. and continue to be seen as a definite identity marker for Central Americans» (p. 105). Igualmente, el uso de la variante apicoalveolar por parte de la informante de Manizales puede ser un elemento identitario de su región de origen.

Con respecto a las limitaciones del trabajo, las conclusiones aquí estipuladas son tentativas y, por lo tanto, sujetas a modificaciones en una investigación más amplia, tanto en la población estudiada como en el número de variables sociolingüísticas analizadas, pues en el presente trabajo de investigación solo fue posible estudiar la variable fonológica /s/ y sus variantes en únicamente cuatro hablantes. Debido a estas razones, se invita a seguir con la línea de la anterior investigación, al analizar diferentes variables y al incluir nuevas variaciones regionales del español colombiano en Filadelfia, (Estados Unidos), de ser posible, con cada uno de los dialectos y superdialectos colombianos.

\section{Referencias bibliográficas}

1. Bernal Chávez, J. A. \& Díaz Romero, C. E. (2017). Caracterización panorámica del español hablado en Colombia: Fonología y gramática. Cuadernos de Lingüística Hispánica, 19-37. http://dx.doi.org/10.19053/0121053X

2. Brown, E. K. \& Brown, E. L. (2012). Syllable-Final and Syllable-Initial/s/ reduction in Cali, Colombia: One Variable or Two? En Colombian Varieties of Spanish. Iberoamericana Vervuert.

3. Canfield, D. L. (1981). Spanish Pronunciation in the Americas. The University Press of Chicago.

4. Corredor Tapias, J. (2018). Aproximación a la sociología del lenguaje: Hacia unas consideraciones generales. Cuadernos de Lingüística Hispánica, 31, 59-77. https://doi.org/10.19053/0121053X.n31.2018.7744

5. del Valle, J. (1998). Andalucismo, poligénesis y koineización: Dialectología e ideología. Hispanic Review, 66(2), 131-149. University of Pennsylvania Press. https://doi.org/10.2307/474524

6. Departamento Administrativo Nacional de Estadística - DANE (2019). Información capital. Recuperado de https://www.dane.gov.co/files/varios/informacion-capital-DANE-2019.pdf 
7. Departamento Administrativo Nacional de Estadística - DANE (2019). Lugar de nacimiento, según área de residencia actual. Dirección de Censos y Demografía. Recuperado de https://sitios.dane.gov.co/cnpv/\#!/lugar_nacimiento

8. Díaz Campos, M. (2014). Introducción a la sociolingüística hispánica. John Wiley \& Sons, Inc.

9. Escobar, A. M., \& Potowski, K. (2015). El español de los Estados Unidos. Cambridge University Press.

10. Espejo Olaya, M. B. (2005). El español bogotano: una lengua de prestigio. Revista La Tadeo,

71. Recuperado a partir de https://revistas.utadeo.edu.co/index.php/RLT/article/view/565

11. Espejo Olaya, M. B. (2016). Alofonía de /s/ en Colombia. Lingüística Y Literatura, 69, 99117. Recuperado a partir de https://revistas.udea.edu.co/index.php/lyl/article/view/26560

12. Feagin, C. (2013). Entering the Community Fieldwork. En Chambers, J. K. \& Schilling, N. (Eds.), The Handbook of Language Variation and Change ( $2^{\text {nd }}$ Edition, pp. 19-37). John Wiley \& Sons, Inc.

13. Flórez, L. (1963). El español hablado en Colombia y su Atlas lingüístico. En Thesaurus, Tomo XVIII (Vol. 2). Instituto Caro y Cuervo.

14. Giles, H. \& Ogay, T. (2007). Communication Accommodation Theory. En B. B. Whaley \& W. Samter (Eds.), Explaining Communication: Contemporary theories and exemplars (pp. 293310). Lawrance Erlbaum.

15. Hualde, J. I., Olarrea, A., Escobar, A. M. \& Catherine E., T. (2010). Introducción a la lingüística hispánica (2a edición). Cambridge University Press.

16. Johnson, E. G. (2005). Mexiqueño? A Case Study of Dialect Contact. University of Pennsylvania Working Papers in Linguistics, 11(2), 91-104. Recuperado de https://repository.upenn.edu/cgi/viewcontent.cgi article $=1539 \&$ context=pwpl

17. Labov, W. (1972). Sociolingustic Patterns. University of Pennsylvania Press.

18. Lipski, J. M. (2003). La lengua española en los Estados Unidos: Avanza a la vez que retrocede. Revista Española de Lingüística, 33(2), 231-260.

19. Lipski, J. M. (2008). Varieties of Spanish in the United States. Georgetown University Press. 20. López Morales, H. (2009). Introducción: Presencia histórica de lo hispano. En Enciclopedia del español en los Estados Unidos. Anuario del Instituto Cervantes 2008. Santillana. 
21. Markič, J. (2017). Phonetic and phonological aspects of Colombian Spanish. Linguistica, 57, 185-196. https://doi.org/10.4312/linguistica.57.1.185-196

22. Matus-Mendoza, M. de la L. (2004). Assibilation of /-r/ and migration among Mexicans. Language Variation and Change, 16, 17-30.

23. Montes Giraldo, J. J. (1982). El español de Colombia: Propuesta de clasificación dialectal. Thesaurus: Boletín del Instituto Caro y Cuervo, 37(1), 23-92.

24. Mora M., S., Lozano R., M., Ramírez C., R. A., Espejo O., M. B., \& Duarte H, G. E. (2004). Caracterización léxica de los dialectos del español de Colombia según el "ALEC”. Instituto Caro y Cuervo.

25. Moreno Fernández, F. (2006). Sociolingüística del español en Estados Unidos. Liceus.

26. Moreno Fernández, F. (2009). Dialectología hispánica de los Estados Unidos. En Enciclopedia del español en los Estados Unidos. Anuario del Instituto Cervantes 2008. Santillana.

27. Orozco, R. (2018). Spanish in Colombia and New York City: Language Contact Meets Dialectal Convergence. John Benjamins.

28. Orozco, R., \& File-Muriel, R. J. (2012). Colombian Spanish at the Turn of the $21^{\text {st }}$ Century. En Colombian Varieties of Spanish. Iberoamericana Vervuert.

29. Otheguy, R., Zentella, A. C., \& Livert, D. (2007). Language and Dialect Contact in Spanish in New York: Toward the Formation of a Speech Community. Language, 38(4), 770-802. https://doi.org/10.1353/lan.2008.0019

30. Ramírez, A., \& Almira, A. (2016). Variación del fonema /s/ en contextos de juntura de palabra en el español caleño: Una mirada sociolingüística. Lenguaje, 44(1), 11-33.

31. Rivera-Mills, S. (2011). Use of Voseo and Latino Identity: An Intergenerational Study of Hondurans and Salvadorans in the Western Region of the U.S. En Selected Proceedings of the $13^{\text {th }}$ Hispanic Linguistics Symposium. Cascadilla Proceedings Project.

32. Silva-Corvalán, C. (2000). La situación del español en Estados Unidos. El español en el mundo. Plaza \& Janés: Círculo de Lectores.

33. Silva-Corvalán, C. (2001). Sociolingüística y pragmática del español. Georgetown University Press. Retrieved from https://lintres.files.wordpress.com/2013/06/carmen-silvacorvalc3a1nsociolingc3bcc3adstica-y-pragmc3a1tica-del-espac3b1ol.pdf 
UN ACERCAMIENTO SOCIOFONOLÓGICO DEL ESPAÑOL COLOMBIANO EN FILADELFIA, ESTADOS UNIDOS: EL CASO DE LA VARIABLE FONOLÓGICA /S/ EN CUATRO HABLANTES DE DIFERENTES REGIONES DEL PAÍS

34. Torres Torres, A. (2010). El español de América en los Estados Unidos. La lengua española en América: Normas y usos actuales. Universitat de València. 